\title{
Inguinal Mass
}

National Cancer Institute

\section{Source}

National Cancer Institute. Inguinal Mass. NCI Thesaurus. Code C35293.

An abnormal growth in the groin. 